\title{
Over-the-Counter Tooth Whitening Agents: A Review of Literature
}

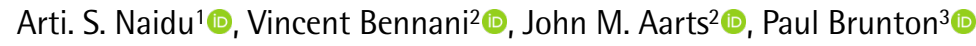

There is an increased accessibility of over-the-counter (OTC) whitening agents with very little data in the literature regarding their effectiveness. This review was done to determine their effectiveness of the predominant OTC whitening agents from 2006 until 2018 where a comparison of each agent was made with a placebo, no treatment or with other OTC whitening agents. The major categories of OTC whitening agents such as dentifrices, whitening strips and paint on gels. Dentist prescribed bleaching applied at home and in-office bleaching studies and studies that demonstrated whitening products to participants were excluded. Articles were searched for in the databases of Medline (Ovid), PubMed, the Cochrane Library and Cochrane Central Register of Controlled Trials. Twenty-four articles were included in the systematic review and the quality of studies was determined by the GRADE (Grading of Recommendations, Assessment, Development and Evaluations) ranking criteria. Compared to other OTC, strips are reported to be effective. Two studies determined whitening strips to be effective. Whitening strips have been shown to be effective when compared with placebos and other OTC whitening agents. Dentifrices are effective in changing the shade of the tooth "by removing extrinsic stains" when compared to a placebo and non-whitening dentifrices, but they are not as effective in comparison to whitening strips. There is a lack of evidence with regards to the effectiveness of paint-on gels. While there is some evidence that OTC can alter shade in the short term, there is a need for better-designed studies.

\author{
'Department of Oral Rehabilitation, \\ School of Dentistry and Oral Health, \\ Fiji National University, Fiji \\ ${ }^{2}$ Department of Oral Rehabilitation, \\ Faculty of Dentistry, University of \\ Otago, Dunedin, New Zealand \\ ${ }^{3}$ Division of Health Sciences, \\ University of Otago, \\ Dunedin, New Zealand
}

Correspondence: Dr Vincent Bennani, PO Box 647, 9054 Dunedin, New Zealand. Tel: +643-479 7000. e-mail: vincent. bennani@stonebow.otago.ac.nz

Key Words: teeth bleaching, teeth whitening, over-the-counter.

\section{Introduction}

In the developed world patients are placing a stronger interest on the aesthetic appearance of their teeth. This paradigm shift coupled with increased accessibility to overthe-counter (OTC) has resulted in an explosion of products marketed directly to the public that claim to address tooth discoloration. Tooth whitening agents aim to remove stains and discolorations. The success of tooth whitening depends on the type of stain present which can be categorized as extrinsic staining, intrinsic staining and internalized discoloration (1). So called "tooth whitening" is a term widely used to describe a change in tooth shade in OTC products marketed to the public. However, the term "tooth whitening" really only describing the end result, while "bleaching" is actually the action that changes the shade of the tooth internally and externally. As OTC products are commonly referred to as "tooth whitening" this term is generically used to describe a change in the tooth shade and the term "tooth whitening agents" refers to the whitening product.

These OTC tooth whitening techniques and materials can easily be applied at-home by the patient. The main difference between at-home teeth whitening and that which are provided / done at the dental practice (in-office) is that athome tooth whitening involves the patient solely purchasing an OTC whitening agent and applying it independently. Another defining difference between OTC whitening and in-office is that the patient makes their own diagnosis and decides on which tooth whitening agent/s to use without any diagnosis of the discoloration (2). In numerous countries another factor is the concentration of hydrogen peroxide or carbamide peroxide (1). The percentage of bleaching agent dictates whether it can be sold direct to the public or not. The amount of hydrogen peroxide or carbamide peroxide available to the public varies depending on the country and the respective regulating authority. Therefore, dentist prescribed tray-based bleaching applied at home and in-office bleaching studies and studies that demonstrated whitening products to participants were excluded from this review.

There are numerous tooth whitening products available, such as, dentifrices, mouth rinses, strips, whitening dental floss, toothbrushes, and paint-on gels or film activated charcoal, oil pulling, etc. However, this review focuses on four major categories of OTC whitening agents, whitening dentifrices, whitening strips, whitening mouth rinses and paint of gels / vanishes. Whitening dentifrice is the most common type of OTC tooth whitening agent found, which contains abrasives for stain removal in conjunction with low amounts of hydrogen peroxide and carbamide peroxide (3). Whitening strips were developed mainly to avoid using customized trays and to allow direct utilization from the public. They typically contain hydrogen peroxide ranging from 5-14\%, which is applied to teeth, and the active ingredients are released 
between 5-60 min once or twice daily (4). Whitening mouth rinses typically contain low concentrations of hydrogen peroxide $(1.5 \%)$ and sodium hexametaphosphate (4. Painton gels or varnishes contain either hydrogen peroxide or carbamide peroxide that adheres to the tooth enamel, and is applied via an applicator onto the tooth surface (5).

There have been reviews that have looked at in office whitening agents and techniques. The last systematic review in 2006 on at-home bleaching products found that all the agents in comparison to placebo/no treatment demonstrated effectiveness. However, the studies included in that review focused only on 2 OTC agents (paint-on gels, and whitening strips) (6). This review investigates the effectiveness of the four major OTC tooth-whitening agents available today. This will be of value to dental practitioners, and will also be informative for the general public.

\section{Material and Methods}

This review was performed in accordance with the PICO approach (Patient or Population, Intervention, Control or Comparison, Outcome, and Study types). The PICO question was formulated as follows: Patient population $(\mathrm{P})=$ General Public, Intervention (I) = Efficacy of OTC agents, Comparison $\dot{5}$ (C) = Efficacy to be compared to dentist prescribed gels, Outcomes (0) = Individual agents efficacy.

A review of the literature published on OTC tooth whitening agents from 2006 until September 2018 was conducted. A search was carried out within Medline (Ovid), PubMed, the Cochrane Library and Cochrane Central Register of Controlled Trials databases. Initially Medline was used to search for articles and a further search in the other databases was used to capture articles not discovered in Medline.

The key words used in Medline (OVID) database were as follows: bleach, bleaching products, tooth, teeth, at home, over the counter, non-prescription, whitening, effects, efficacy, effectiveness, toothpastes, strips, mouth rinses, paint on films, dentifrices, bleaching. The key words were also searched in multiple combinations. The key words used in PubMed database were as follows: over the counter OR at home OR non-prescribed AND efficiency OR effectiveness AND tooth bleaching OR teeth bleaching OR tooth whitening OR teeth whitening. The key words used in Cochrane Library and Cochrane Central Register of Controlled Trials database were as follows: bleach $\mathrm{OR}$ bleaching products $\mathrm{OR}$ whitening $\mathrm{OR}$ toothpastes $O R$ dentifrices $O R$ strips $O R$ mouth rinse $O R$ paint on AND at home OR over the counter AND tooth OR teeth.

The following inclusion and exclusion criteria aimed to include clinical studies done with OTC tooth whitening agents including both chemical and mechanical based agents. Therefore, this review was not confined to randomized controlled clinical studies only. The criteria were made to reflect the whitening agents that are readily available over the counter without any professional guidance. The inclusion criteria included all clinical studies involving OTC tooth whitening agents (dentifrices, whitening strips, mouth rinses, and paint-on gels/varnishes) from 2006 to September 2018. Studies comparing whitening agents to a placebo or comparing to other whitening agents were also included. Studies that had adults aged 18 years and over were included as they would be the population choosing such agents and applying it independently. The exclusion criteria included studies involving participants of younger than 18 years of age and non-English studies. Dentist prescribed tray-based bleaching kits applied at home and in-office bleaching studies and studies that demonstrated whitening products to participants were excluded. In-vitro studies were excluded as this review aims to provide evidence for making clinical decisions. Whitening chewing gum essentially contain sodium hexametaphosphate (4.0-7.5\%) and are promoted to prevent extrinsic tooth stain formation (6), as they are predominately reported to be associated with preventing stain formation they have not been included in the review.

Eight hundred and twelve studies between 2006 and September 2018 were initially identified by the above search parameters. According to the titles, 118 studies were identified as relevant and abstracts retrieved for evaluation. Full text articles were retrieved when further information was required to determine suitability of the study for inclusion. Inclusion and exclusion criteria were applied, which resulted in 24 studies being identified for the final review (Fig. 1).

To identify all information relevant to this review study, the principal researcher followed a two-stage literature search strategy. First, an intensive electronic search of Englishlanguage literature published between January 1, 2006, and September 30, 2018. One reviewer verified the eligibility of the potentially relevant articles and independently screened titles and abstracts to evaluate the articles for full-text reading. When the abstract was deficient, titles were used to obtain full texts, and consensus was achieved after discussion with another reviewer. If they disagreed a third reviewer was consulted. In the second round of the search, only studies meeting specific inclusion criteria were selected. Finally, to supplement the electronic search, a hand search was conducted by considering entries in the reference lists of the selected articles, and articles not yet included were then added.

The studies were classified based on the type of tooth whitening agents being trialed such as dentifrices, whitening strips, mouth rinses, and paint-on gels/varnishes. A category was added that grouped all the clinical studies that looked at one or more 0TC tooth-whitening agent being trialed. Studies selected were either trialed in comparison with a placebo, no treatment, and dentist-prescribed tray-based gels or with other OTC tooth whitening agents. After the classification into different groups of agents, analysis of full text articles 
was carried out by looking into detail at the aim of the study and the methodology carried. The studies were ranked using the GRADE system (British Medical Journal clinical evidence for assessing the quality of studies) to assess the risk of bias $(7,8)$. Studies that were ranked with a score of 3 and above were classified as good quality. Whilst ranking the studies, the critical areas in methodology such as randomization, blinding, process of inclusion of participants, preparation of participants or specimens, consistency in process of shade taking before and after tooth whitening procedures, determined the GRADE ranking. Results and conclusion of each study were reported. After identifying each of the categories above and ranking each study, good quality studies were further reviewed to ascertain the effectiveness of the tooth whitening agents investigated. To determine the effectiveness of each agent all of the included studies' conclusions were considered, along with the quality of the studies, with the higher quality studies ultimately being the main contributing factor. The present study was conducted and reported adhering to the PRISMA (Preferred Reporting Items for Systematic Review and Meta-Analyses) statement (9).

\section{Results}

From the 24 studies included in this review, 22 randomized clinical studies, 1 was a randomized double-blind study, and 1 was a clinical study. Ten studies were on whitening dentifrices, 7 on whitening strip, and 2 on paint-on gels/ varnish. No in vivo studies were identified relating to the whitening mouth rinses. Five studies compared two or more OTC agents (Boxes 1-3).

With regards to shade analysis, 11 studies used digital

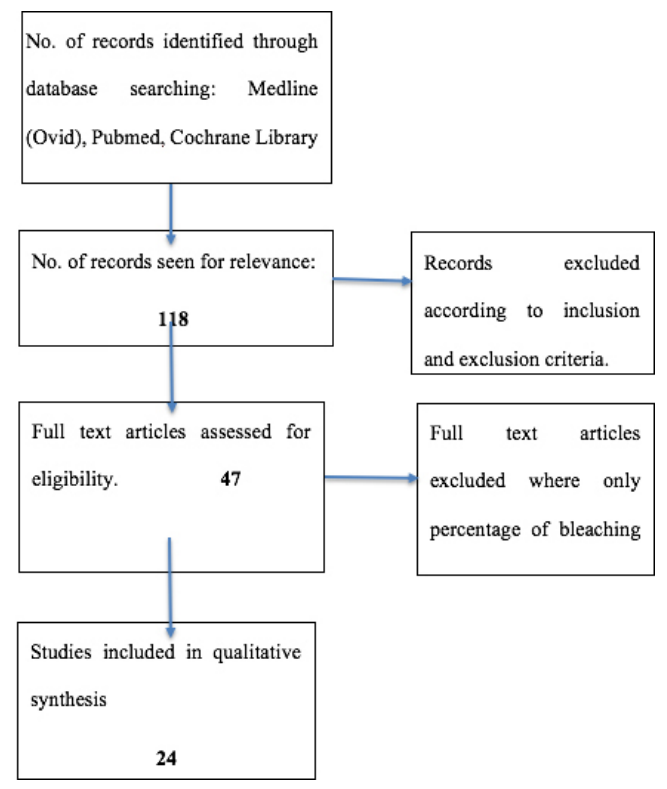

Figure 1. Process of identifying studies. imaging, 1 used digital imaging in conjunction with a shade guide and 1 used digital imaging in conjunction with the WIO: Whiteness formula index. Five studies used a VITA Classic shade guide and 5 used the Lobene Stain Index or a Modified Lobene Stain Index (Boxes 1-4). The studies observational periods were not consistent with only one study having a period more than a year. The age range of participants was from 18 to 77 .

The largest numbers of studies were related to whitening dentifrice, which included 8 randomized clinical study, 1 randomized double-blind clinical study, 1 controlled crossover clinical study, and 1 clinical study (Box 1). The tooth whitening elements varied considerably amongst studies. Tooth whitening protocols involved brushing from 1 to 3 min twice daily. The quality of the studies ranged from GRADE $0-3$, with 2 studies of good quality reporting that dentifrice was effective in changing the shade of the tooth (via stain removal) (Box 1) $(10,11)$, but had lower effectiveness when compared to whitening strip as shown in Box 4 (12).

There were 2 good quality studies that investigated whitening strips, which determined that whitening strips were effective (Box 2) $(13,14)$, and were also effective when compared to other tooth whitening agents $(12,15-17)$. There were 7 studies on whitening strips included, all 7 were randomized clinical studies (Box 2). The sample size was below 50 in all these studies and all the studies reported significant change. The main whitening agent used was hydrogen peroxide with concentration ranging from 5 to $10 \%$. The application protocol was twice daily for $30 \mathrm{~min}$, which varied from 14 days to 6 weeks.

There were no good quality studies in relation to paint-on gels, as shown in Box 3. However, in Box 4, there are 3 good quality studies that compared paint-on gels with whitening strips and all reported that whitening strips were more effective than paint-on gels $(15,17,18)$. There were 2 studies that trailed paint-on gels/vanishes; these were randomized clinical studies and both had a GRADE 2 (Box 3) $(19,20)$. The main whitening agent used for paint-on gels/varnish was hydrogen peroxide, which was in different concentrations in the studies. Paint-on agents were of a gel or varnish medium. The protocols differed between the two studies, ranging from the varnish being applied for 10 min for 5 days to the gel being applied for 15 min for 14 days.

Five studies compared two or more OTC whitening agents (Box 4). There were 4 randomized clinical studies and 1 clinical study. There were different agents with different tooth whitening protocols. The quality ranged was predominantly good, with 4 out of the 5 studies having a GRADE score of 3 . All studies that included whitening strips, determined that whitening strips were more effective than the other OTC whitening agents, such as dentifrices and paint-on-gels. 
Box 1. Description of whitening dentifrice clinical studies

\begin{tabular}{|c|c|c|c|c|c|}
\hline Authors & Study design & $\mathrm{n}$ & Whitening agent & Whitening protocol & $\begin{array}{c}\text { Shade } \\
\text { appraisal }\end{array}$ \\
\hline \multirow{2}{*}{$\begin{array}{l}\text { Collins et } \\
\text { al., } 2008\end{array}$} & \multirow{2}{*}{$\begin{array}{l}\text { Controlled } \\
\text { cross-over } \\
\text { clinical study }\end{array}$} & \multirow[t]{2}{*}{78} & Group 1: Blue covarine & $\begin{array}{l}\text { Group } 1 \text { Brush for } 1.5 \mathrm{~min} \\
\text { twice daily for } 1 \text { day }\end{array}$ & \multirow[t]{2}{*}{ S WIO Index } \\
\hline & & & Group 2: Placebo (Clear gel) & $\begin{array}{l}\text { Group 2: Brush for } 1.5 \mathrm{~min} \\
\text { twice daily for one day }\end{array}$ & \\
\hline $\begin{array}{l}\text { Forner et } \\
\text { al., } 2012\end{array}$ & Clinical study & 20 & $5 \%$ lactoperoxidase $+3 \%$ carbamide peroxide & $\begin{array}{l}\text { Brush for } 3-4 \min 3 \\
\text { times daily for } 21 \text { days }\end{array}$ & $\mathrm{S}$ \\
\hline \multirow{3}{*}{$\begin{array}{l}\text { Ghassemi, } \\
2015\end{array}$} & \multirow{3}{*}{$\begin{array}{l}\text { Randomized } \\
\text { controlled } \\
\text { study }\end{array}$} & & $\begin{array}{c}\text { Group 1: 35\% sodium bicarbonate, sodium fluoride, } \\
\text { Amorphous calcium phosphate (Truly radiant } \\
\text { toothpaste) }\end{array}$ & $\begin{array}{l}\text { Group } 1 \text { :Brush for } 2 \text { min } \\
\text { twice daily for } 6 \text { weeks }\end{array}$ & \multirow{3}{*}{ VCS } \\
\hline & & 178 & $\begin{array}{l}\text { Group 2: Crest 3D whitening } \\
\text { toothpaste (positive control) }\end{array}$ & $\begin{array}{l}\text { Group 2: Brush for } 2 \text { min } \\
\text { twice daily for } 6 \text { weeks }\end{array}$ & \\
\hline & & & Group 3: Colgate cavity protection (negative control) & $\begin{array}{l}\text { Group 3: Brush for } 2 \text { min } \\
\text { twice daily for } 6 \text { weeks }\end{array}$ & \\
\hline \multirow{2}{*}{$\begin{array}{l}\text { Ghassemi, } \\
2012\end{array}$} & \multirow{2}{*}{$\begin{array}{l}\text { Randomized } \\
\text { controlled } \\
\text { study }\end{array}$} & \multirow[t]{2}{*}{135} & Group 1: Sodium bicarbonate & $\begin{array}{l}\text { Group 1: Brush for } 1 \text { min } \\
\text { twice daily for } 6 \text { weeks }\end{array}$ & \multirow[t]{2}{*}{ VCS } \\
\hline & & & Group 2: Placebo (silica based) & $\begin{array}{l}\text { Group 2: Brush for } 1 \text { min } \\
\text { twice daily for } 6 \text { weeks }\end{array}$ & \\
\hline \multirow{2}{*}{$\begin{array}{l}\text { Ghassemi } \\
\text { et al., } 2015\end{array}$} & \multirow{2}{*}{$\begin{array}{l}\text { Randomized } \\
\text { controlled } \\
\text { study }\end{array}$} & \multirow[t]{2}{*}{54} & $\begin{array}{l}\text { Group 1:20\% sodium bicarbonate, sodium } \\
\text { fluoride, tetrasodium pyrophosphate (Truly } \\
\text { Radiant Rejuvenating toothpaste) }\end{array}$ & $\begin{array}{l}\text { Group 1: Brush for } 2 \text { min } \\
\text { twice daily for } 5 \text { days }\end{array}$ & \multirow[t]{2}{*}{ LSI } \\
\hline & & & Group 2: Colgate cavity protection (negative control) & $\begin{array}{l}\text { Group 2: Brush for } 2 \text { min } \\
\text { twice daily for } 5 \text { days }\end{array}$ & \\
\hline \multirow{4}{*}{$\begin{array}{l}\text { Horn et } \\
\text { al., } 2014\end{array}$} & \multirow{4}{*}{$\begin{array}{l}\text { Randomized } \\
\text { clinical study }\end{array}$} & \multirow{4}{*}{60} & $\begin{array}{l}\text { Group 1: Water, hydrated silica, sodium } \\
\text { lauryl sulphate, sodium hydroxide, sodium } \\
\text { fluoride, Triclosan, sodium saccharin, } \\
\text { titanium dioxide (Colgate Total 12) }\end{array}$ & & \multirow{4}{*}{$\mathrm{S}$} \\
\hline & & & $\begin{array}{l}\text { Group 2: Water, sorbitol, hydrated silica, } \\
\text { sodium lauryl sulphate, sodium fluoride, } \\
\text { sodium saccharin, trisodium phosphate, } \\
\text { dipentene (Close Up White Now) }\end{array}$ & Groups 1- 4: Brush & \\
\hline & & & $\begin{array}{l}\text { Group 3: Sodium fluoride, water, hydrated } \\
\text { silica, sorbitol, sodium lauryl sulphate, sodium } \\
\text { hydroxide, sodium saccharin (Oral B 3D White) }\end{array}$ & $\begin{array}{l}\text { for 2-3 min, } 3 \text { times } \\
\text { daily for } 15 \text { days }\end{array}$ & \\
\hline & & & $\begin{array}{l}\text { Group 4: Water, hydrated silica, sorbitol, } \\
\text { pentasodium triphosphate, tetrapotassium } \\
\text { pyrophosphate, sodium lauryl sulfate, polyethylene, } \\
\text { cocamidopropyl betaine, sodium saccharin, } \\
\text { sodiumhydroxide, sodium fluoride, titanium dioxide, } \\
\text { blue \# } 1 \text { aluminium lac (Colgate Luminous White) }\end{array}$ & & \\
\hline \multirow{3}{*}{$\begin{array}{l}\text { Nathoo et } \\
\text { al., } 2008\end{array}$} & \multirow{3}{*}{$\begin{array}{l}\text { Randomized } \\
\text { clinical study }\end{array}$} & $\begin{array}{l}\text { Study } \\
1: 114\end{array}$ & $\begin{array}{c}\text { Studies } 1 \text { and } 2 \\
\text { Group 1: } 0.3 \% \text { triclosan/ 2.0\% PVM/MA } \\
\text { copolymer/0.243\% sodium fluoride in a 17\% } \\
\text { dual silica base (Colgate Total Advanced) }\end{array}$ & $\begin{array}{l}\text { Studies } 1 \text { and } 2 \\
\text { Group 1: Brush for } 1 \mathrm{~min} \\
\text { twice daily for } 6 \text { weeks }\end{array}$ & \multirow{3}{*}{ LSI } \\
\hline & & & $\begin{array}{l}\text { Group 2: } 0.243 \% \text { sodium fluoride in a } \\
\text { silica base (Crest Cavity Protection) }\end{array}$ & $\begin{array}{l}\text { Group 2: Brush for } 1 \mathrm{~min} \\
\text { twice daily for } 6 \text { weeks }\end{array}$ & \\
\hline & & $\begin{array}{l}\text { Study } \\
\text { 2: } 119\end{array}$ & & & \\
\hline
\end{tabular}




\begin{tabular}{|c|c|c|c|c|c|c|c|}
\hline \multicolumn{7}{|c|}{ Shade change $\mathrm{x}$ days after treatment } & \multirow{2}{*}{$\begin{array}{l}\text { GRADE } \\
\text { score }\end{array}$} \\
\hline Baseline & 0 days & 5 days & 2 weeks & 3 weeks & 6 weeks & 8 weeks & \\
\hline $\begin{array}{l}\text { Group 1 WIO: } \\
\text {-55.28 (1.70) }\end{array}$ & $\begin{array}{c}\text { Group 1 } \Delta \text { WIO: } \\
1.14(0.30) \\
(\mathrm{p}<0.05)\end{array}$ & & & & & & 0 \\
\hline $\begin{array}{c}\text { Group 2: WIO: } \\
\text {-55.03(1.71) }\end{array}$ & $\begin{array}{c}\text { Group 2: } \Delta \text { WIO: } \\
0.08(0.31)\end{array}$ & & & & & & \\
\hline $\begin{array}{l}\text { Shade B2 } \\
\text { or darker }\end{array}$ & & & & $\Delta \mathrm{E}: 5.14$ & & & 0 \\
\hline $\begin{array}{c}\text { Group 1: VCS: } \\
8.981 \text { (2.57) }\end{array}$ & & & & & $\begin{array}{c}\text { Group 1: } \triangle \text { VCS: } \\
-2.08(\mathrm{p}<0.05)\end{array}$ & & \\
\hline $\begin{array}{c}\text { Group 2: VCS: } \\
8.969(2.39)\end{array}$ & & & & & $\begin{array}{l}\text { Group 2: } \triangle \text { VCS: } \\
-1.467(\mathrm{p}<0.05)\end{array}$ & & 0 \\
\hline $\begin{array}{c}\text { Group 3: VCS: } \\
9.079 \text { (2.78) }\end{array}$ & & & & & $\begin{array}{c}\text { Group 3: } \\
\Delta \text { VCS: }-0.038 \\
(\mathrm{p}=0.6713)\end{array}$ & & \\
\hline $\begin{array}{c}\text { Group 1: VCS: } \\
9.77(2.26)\end{array}$ & & & & & $\begin{array}{l}\text { Group 1: } \triangle \text { VCS: } \\
-2.57 \text { (0.99) }\end{array}$ & & 1 \\
\hline $\begin{array}{c}\text { Group 2: VCS: } \\
9.17(2.14)\end{array}$ & & & & & $\begin{array}{l}\text { Group 2: } \triangle \mathrm{VCS}: \\
-0.04(0.69)\end{array}$ & & \\
\hline $\begin{array}{l}\text { Group 1: LSI: } \\
3.094 \text { (0.99) }\end{array}$ & & $\begin{array}{c}\text { Group 1: } \Delta \text { LSI: } \\
2.38 \text { (0.99) }\end{array}$ & & & & & 2 \\
\hline $\begin{array}{l}\text { Group 2: LSI: } \\
3.273 \text { (1.79) }\end{array}$ & & $\begin{array}{c}\text { Group 2: } \Delta \text { LSI: } \\
3.32(1.80)\end{array}$ & & & & & \\
\hline Group 1: L: 82.9 & & & $\begin{array}{l}\text { Group 1: } \\
\Delta \mathrm{E}:-0.25\end{array}$ & & & & \\
\hline Group 2: L: 83.9 & & & $\begin{array}{l}\text { Group 2: } \\
\Delta \mathrm{E}:-0.48\end{array}$ & & & & \\
\hline Group 3: L: 83.9 & & & $\begin{array}{l}\text { Group 3: } \\
\Delta \mathrm{E}: 0.29\end{array}$ & & & & 1 \\
\hline Group 4: L: 86.4 & & & $\begin{array}{l}\text { Group 4: } \\
\Delta \mathrm{E}: 1.15\end{array}$ & & & & \\
\hline $\begin{array}{l}\text { Study } 1 \text { Group 1: } \\
\text { LSI: } 0.68 \pm 0.21\end{array}$ & & & & & $\begin{array}{c}\text { Study } 1 \text { Group } \\
\text { 1: } \Delta \text { LSI: - } \\
0.12 \pm 0.11\end{array}$ & & \\
\hline $\begin{array}{c}\text { Group 2: LSI } \\
0.70 \pm 0.17\end{array}$ & & & & & $\begin{array}{c}\text { Group 2: } \Delta \text { LSI: } \\
0.25 \pm 0.16\end{array}$ & & 0 \\
\hline $\begin{array}{l}\text { Study } 2 \text { Group } 1: \\
\text { LSI: } 1.30 \pm 0.25\end{array}$ & & & & & $\begin{array}{c}\text { Study } 2 \text { Group } \\
1: \Delta \text { LSI :- } \\
0.79 \pm 0.36\end{array}$ & & \\
\hline $\begin{array}{c}\text { Group 2: LSI: } \\
1.6 \pm 0.23\end{array}$ & & & & & $\begin{array}{l}\text { Group 2: } \Delta \text { LSI: } \\
-1.52 \pm 0.48\end{array}$ & & \\
\hline
\end{tabular}




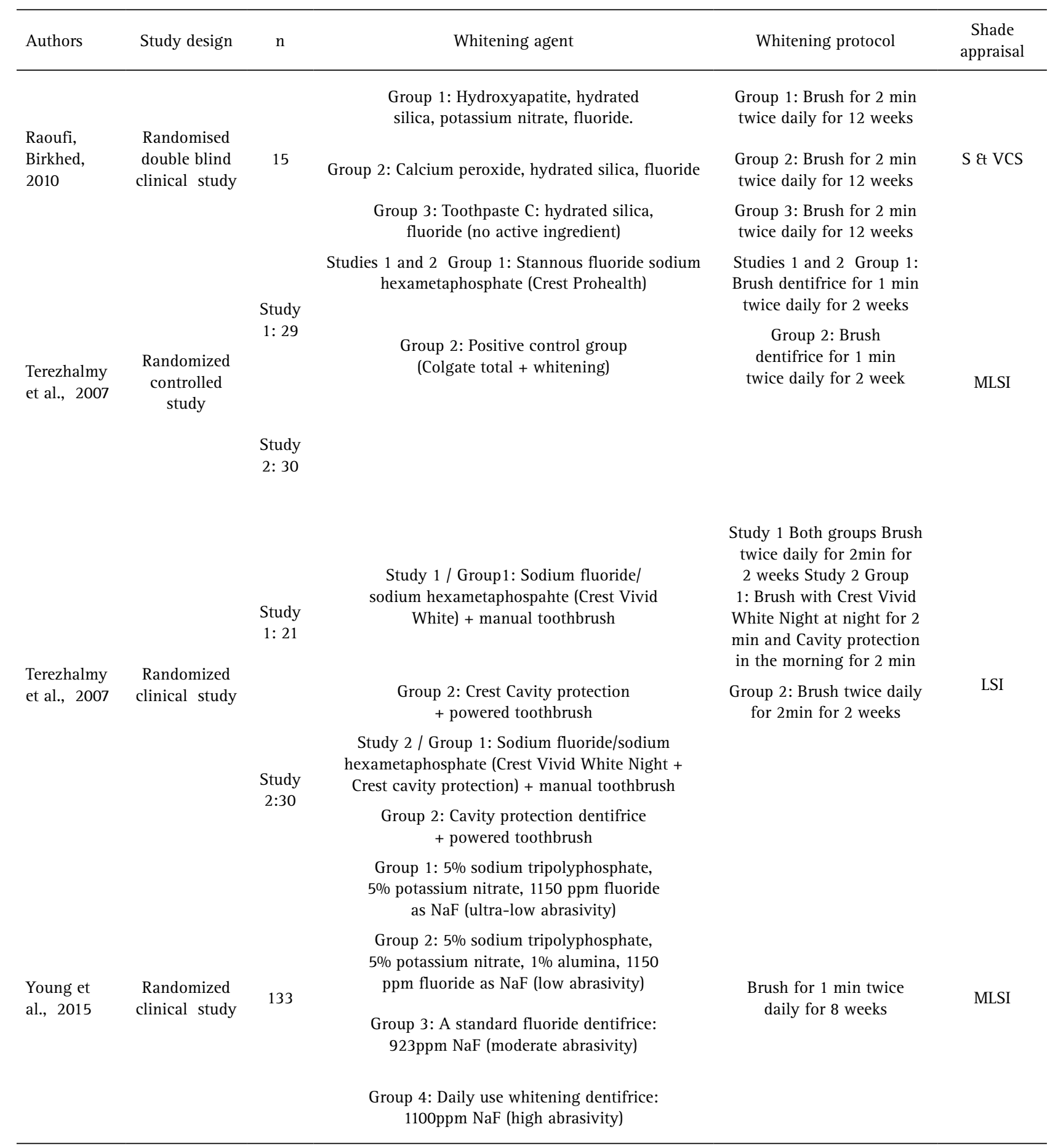




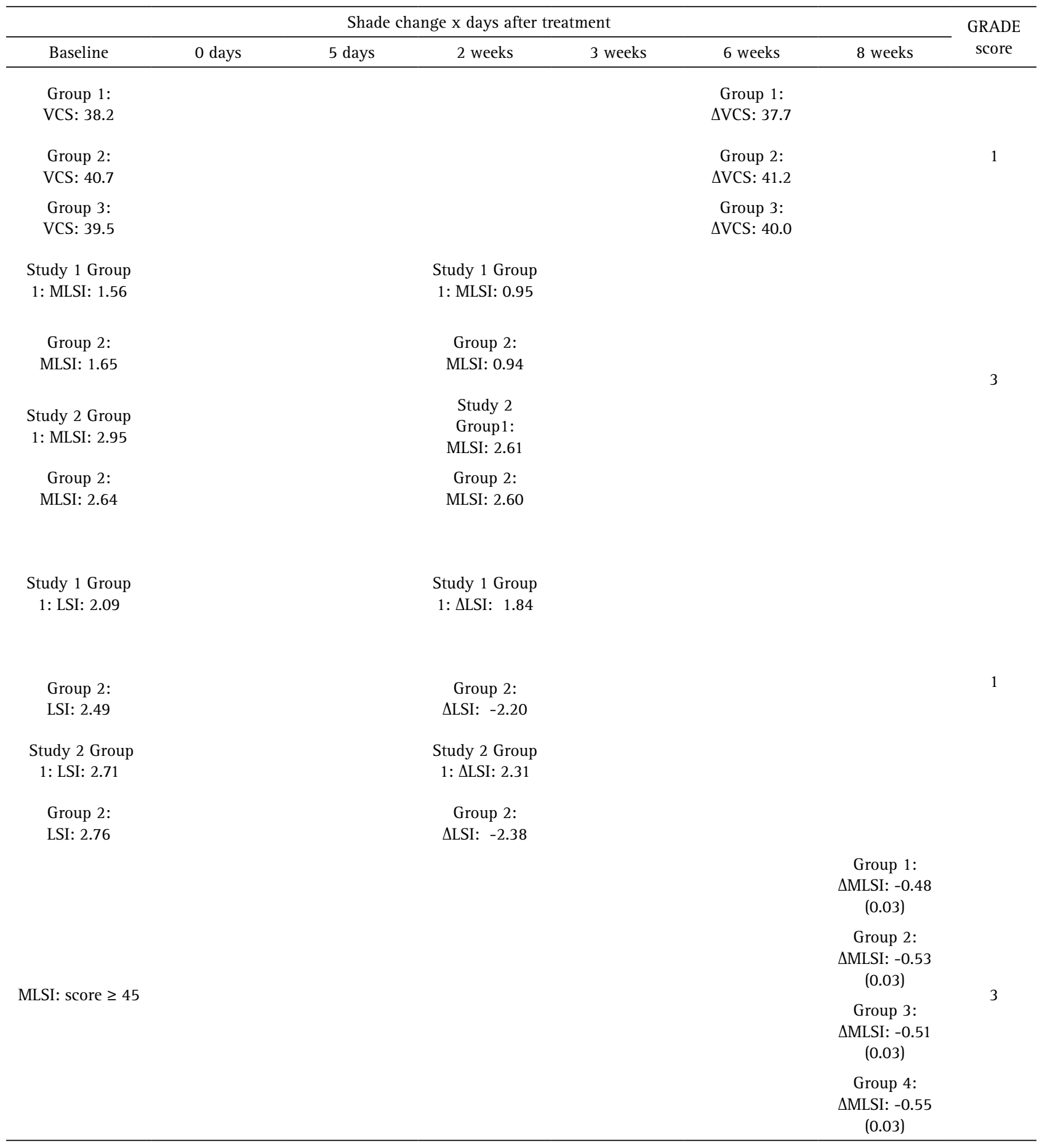

NaF: Sodium fluoride. WIO: Whiteness formula index. VCS: Vitapan Classical Shade Guide. LSI: *Lobene Stain Index. S: Spectrophotometer. MLSI: Modified Lobene Stain Index. L: Lightness. $\triangle$ WIO: Changes in whiteness of teeth according to WIO index. $\Delta$ VCS: Change in tooth shade according to VCS. $\triangle$ LSI: Change in tooth shade according to LSI. $\Delta \mathrm{E}$ : Color difference before and after treatment according to S. $\Delta$ MLSI: Change in tooth shade according to MLSI.

\begin{tabular}{lcc}
\hline${ }^{*}$ Lobene Stain Index Score & Intensity Description & Area Description \\
\hline 0 & No Stain & No Stain \\
1 & Light Stain (yellow to light brown or grey) & Stain covering up to $1 / 3$ of the region \\
2 & Moderate Stain (medium brown) & Stain covering $1 / 3$ up to $2 / 3$ of the region \\
3 & Heavy Stain (dark brown to black) & Stain covering $>2 / 3$ of the region \\
\hline
\end{tabular}


Box 2. Description of whitening strips clinical studies

\begin{tabular}{|c|c|c|c|c|c|}
\hline Authors & Study Design & $\mathrm{n}$ & Whitening Agent & Whitening Protocol & $\begin{array}{c}\text { Color } \\
\text { Appraisal }\end{array}$ \\
\hline \multirow{3}{*}{$\begin{array}{l}\text { Auschill } \\
\text { et al., } 2012\end{array}$} & & \multirow{3}{*}{28} & $\begin{array}{c}\text { Group 1: } \\
\text { 5.3\% HP strips }\end{array}$ & $\begin{array}{c}\text { Group 1: } \\
\text { Twice daily for } 30 \\
\text { min for } 2 \text { weeks }\end{array}$ & \multirow{3}{*}{ VCS } \\
\hline & $\begin{array}{c}\text { Randomized clinical } \\
\text { study }\end{array}$ & & & & \\
\hline & & & $\begin{array}{l}\text { Group 2: } \\
5.0 \% \text { HP tray } \\
\text { at home }\end{array}$ & $\begin{array}{c}\text { Group 2: } \\
\text { Twice daily for } 30 \\
\text { min for } 2 \text { weeks }\end{array}$ & \\
\hline \multirow{2}{*}{$\begin{array}{l}\text { da Costa } \\
\text { et al., } 2012\end{array}$} & \multirow{2}{*}{$\begin{array}{c}\text { Randomized clinical } \\
\text { study }\end{array}$} & \multirow{2}{*}{24} & $\begin{array}{c}\text { Group 1: } \\
\text { 14\% HP strips }\end{array}$ & $\begin{array}{c}\text { Group 1: } \\
\text { Twice daily for } 30 \\
\text { min for } 2 \text { weeks }\end{array}$ & \multirow{2}{*}{$S$} \\
\hline & & & $\begin{array}{l}\text { Group 2: } \\
\text { 35\% CP tray at home }\end{array}$ & $\begin{array}{c}\text { Group 2: } \\
\text { Twice daily for } 30 \\
\text { min for } 2 \text { weeks }\end{array}$ & \\
\hline \multirow{2}{*}{$\begin{array}{l}\text { Ferrari } \\
\text { et al., } 2007\end{array}$} & \multirow{2}{*}{$\begin{array}{l}\text { Randomized clinical } \\
\text { study }\end{array}$} & \multirow{2}{*}{36} & $\begin{array}{l}\text { Group 1: } \\
\text { 6\% HP strip }\end{array}$ & $\begin{array}{c}\text { Group 1: } \\
\text { Twice daily for } 30 \\
\text { min for } 2 \text { weeks }\end{array}$ & \multirow{2}{*}{$S$} \\
\hline & & & $\begin{array}{l}\text { Group 2: } \\
\text { 10\% CP tray at home }\end{array}$ & $\begin{array}{c}\text { Group 2: } \\
\text { Twice daily for } 30 \\
\text { min for } 2 \text { weeks }\end{array}$ & \\
\hline \multirow{2}{*}{$\begin{array}{l}\text { Hanning } \\
\text { et al., } 2007\end{array}$} & \multirow{2}{*}{$\begin{array}{c}\text { Randomized clinical } \\
\text { study }\end{array}$} & \multirow{2}{*}{47} & $\begin{array}{l}\text { Group 1: } \\
\text { 6\% HP strips }\end{array}$ & $\begin{array}{c}\text { Group 1: } \\
\text { Twice daily for } 30 \\
\text { min for } 2 \text { weeks }\end{array}$ & \multirow{2}{*}{$S$} \\
\hline & & & $\begin{array}{l}\text { Group 2: } \\
\text { 10\% CP tray at home }\end{array}$ & $\begin{array}{l}\text { Group 2: } \\
1 \text { hr daily for } \\
2 \text { weeks }\end{array}$ & \\
\hline \multirow{2}{*}{$\begin{array}{l}\text { Guerrero } \\
\text { et al., } 2007\end{array}$} & \multirow{2}{*}{$\begin{array}{l}\text { Randomized } \\
\text { controlled } \\
\text { study }\end{array}$} & & $\begin{array}{c}\text { Group 1: } \\
6.5 \% \text { HP strips }\end{array}$ & $\begin{array}{c}\text { Group 1: } \\
\text { Twice daily for } 30 \\
\text { min for } 3 \text { weeks }\end{array}$ & \multirow{2}{*}{$S$} \\
\hline & & & $\begin{array}{l}\text { Group 2: } \\
\text { Placebo }\end{array}$ & $\begin{array}{c}\text { Group 2: } \\
\text { Twice daily for } 30 \\
\text { min for } 3 \text { weeks }\end{array}$ & \\
\hline \multirow{3}{*}{$\begin{array}{l}\text { Oliveira } \\
\text { et al., } 2013\end{array}$} & \multirow{3}{*}{$\begin{array}{c}\text { Randomized clinical } \\
\text { study }\end{array}$} & \multirow{3}{*}{39} & $\begin{array}{c}\text { Group 1: } \\
\text { 9.5\% HP strips }\end{array}$ & $\begin{array}{c}\text { Group 1: } \\
2 \text { hrs daily for } 9 \text { days }\end{array}$ & \multirow{3}{*}{$\mathrm{S}$} \\
\hline & & & & & \\
\hline & & & $\begin{array}{l}\text { Group 2: } \\
\text { 10\% HP strips }\end{array}$ & $\begin{array}{l}\text { Group 2: } \\
30 \text { min daily } \\
\text { for } 9 \text { days }\end{array}$ & \\
\hline \multirow{2}{*}{$\begin{array}{l}\text { Swift } \\
\text { et al., } 2009\end{array}$} & \multirow{2}{*}{$\begin{array}{l}\text { Randomized } \\
\text { controlled } \\
\text { study }\end{array}$} & & $\begin{array}{l}\text { Group 1: } \\
\text { 6\% HP strips }\end{array}$ & $\begin{array}{c}\text { Group 1: } \\
\text { Twice daily for } 30 \\
\text { min for } 6 \text { weeks }\end{array}$ & \multirow{2}{*}{$S$} \\
\hline & & & $\begin{array}{c}\text { Group 2: } \\
\text { Placebo strips } \\
\text { (no HP) }\end{array}$ & $\begin{array}{c}\text { Group 2: } \\
\text { Twice daily for } 30 \\
\text { min for } 6 \text { weeks }\end{array}$ & \\
\hline
\end{tabular}




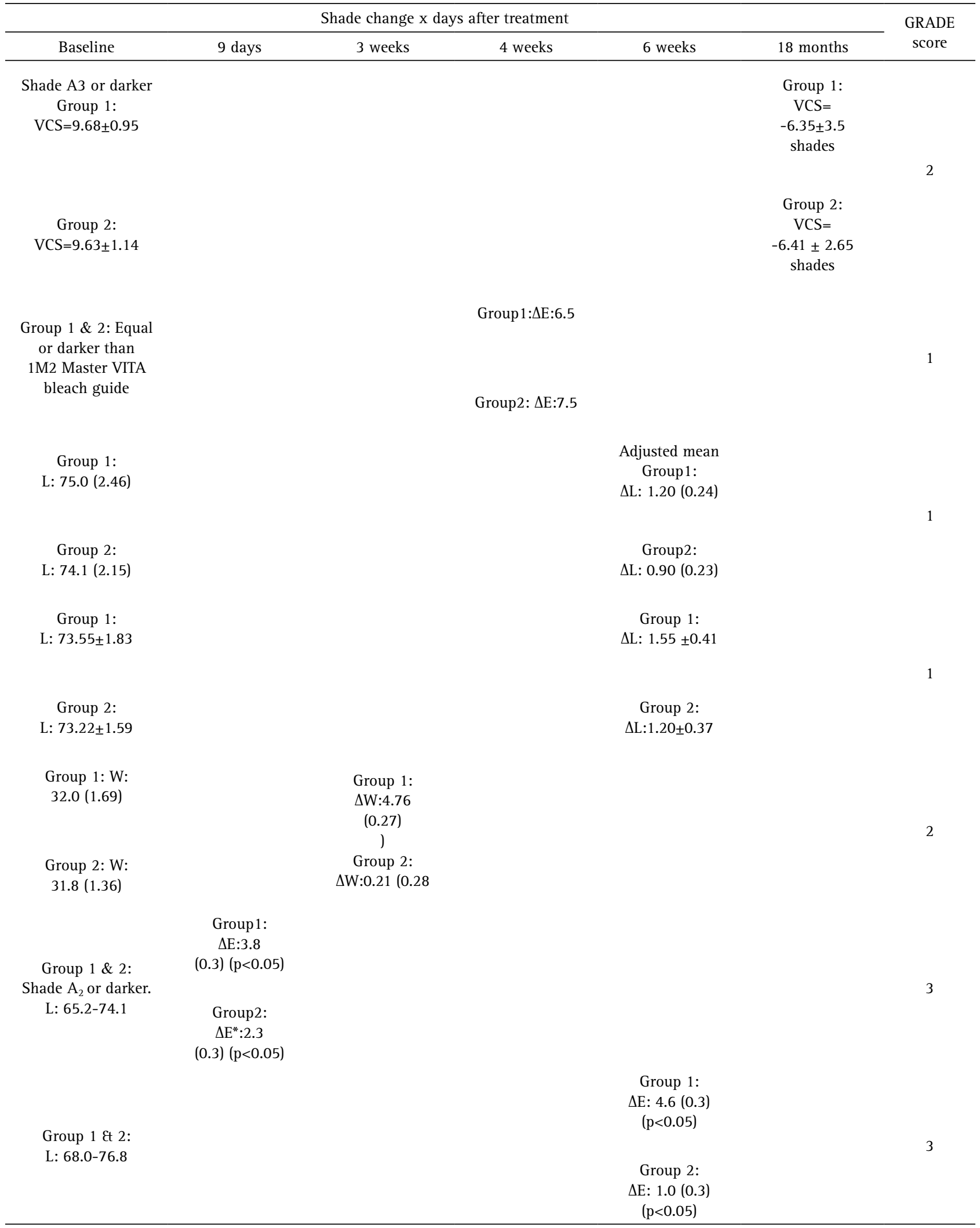

HP: Hydrogen peroxide; CP: Carbamide peroxide; S: Spectrophotometer; $\triangle \mathrm{E}$ : Color difference before and after treatment according to S L: Lightness; $\triangle \mathrm{L}$ : Lightness difference before and after treatment according to S; VCS: Vitapan Classical Shade Guide; VCS: Change in tooth shade according to VCS; W: Whiteness: $\triangle \mathrm{W}$ : Change in the closeness to white. 


\section{Discussion}

This review attempts to determine the effectiveness of major OTC tooth whitening agents currently available. It was challenging to analyze the effectiveness of a particular whitening agent compared to another due to the lack of consistent research protocols. For example, some studies dried the teeth after tooth whitening to assess the shade and others did not or did not specify. This was again inconsistent with regards polishing of teeth and the positioning shade taking device on the arch was specified in some studies, and not others. The temperature of the light source in the room were often not clear and which teeth were measured to assess the bleaching efficiency was often not identified. Also, the color-measuring instrument are not consistent with some studies using conventional color measuring techniques, while others used digital directly or indirectly. Spectrophotometers can determine a color change that cannot be perceived by the human eye; however, it is challenging to take multiple measurements when removing the instrument from the tooth as placing the instrument back in the same location is critical to having consistent readings. Multiple studies used a shade assessment technique/device that were only suitable to $\vec{\sigma}$ determine the change in the surface shade, for example the Lobene stain index measures surface stain removal of only, and not change in tooth color.

In this review, 10 studies used subjective measures (Vita Classic shade guide, Lobene Stain Index and modified Lobene Stain Index), 14 used an objective device (spectrophotometer) and only 1 study used both type of these measures. A review stated that spectrophotometer provides a more consistent comparison of shade change as opposed to conventional shade guides. Conventional shade guides allow a non-linear evaluation of a specific shade without considering hue, value and chroma separately. Specific shade guides such as the Vita 3D Master or digital imaging devices provide a linear evaluation of a shade considering its hue, value, and chroma separately. This highlights an important point when analyzing the results, as different tools would give different levels of accuracy of shade. This brings into question the accuracy of shade measurements and the results of studies that used a VITA Classic shade guide. It has been recommended that the best way to ensure precise shade taking is to use both subjective and objective measures (21).

It could be argued that all OTC studies have an element of bias due to the studies evaluation process and the inclusion and exclusion criterion being applied. An area of possible bias is that a number of studies involved researchers that were employees of the whitening agent manufacturer and the majority of the studies involving whitening strips and dentifrices were either partially or fully funded by the manufacturers. Not every study had standardized patient selection (age, hygiene, baseline color, and diet). Several studies provided instructions or demonstrated how to apply the OTC whitening agent, which could skew the outcomes, as OTC products are provided with no professional advice.

\section{Whitening Dentifrices}

Whitening dentifrices is one of the OTC agents that are

Box 3. Description of paint-on gel / varnish clinical studies

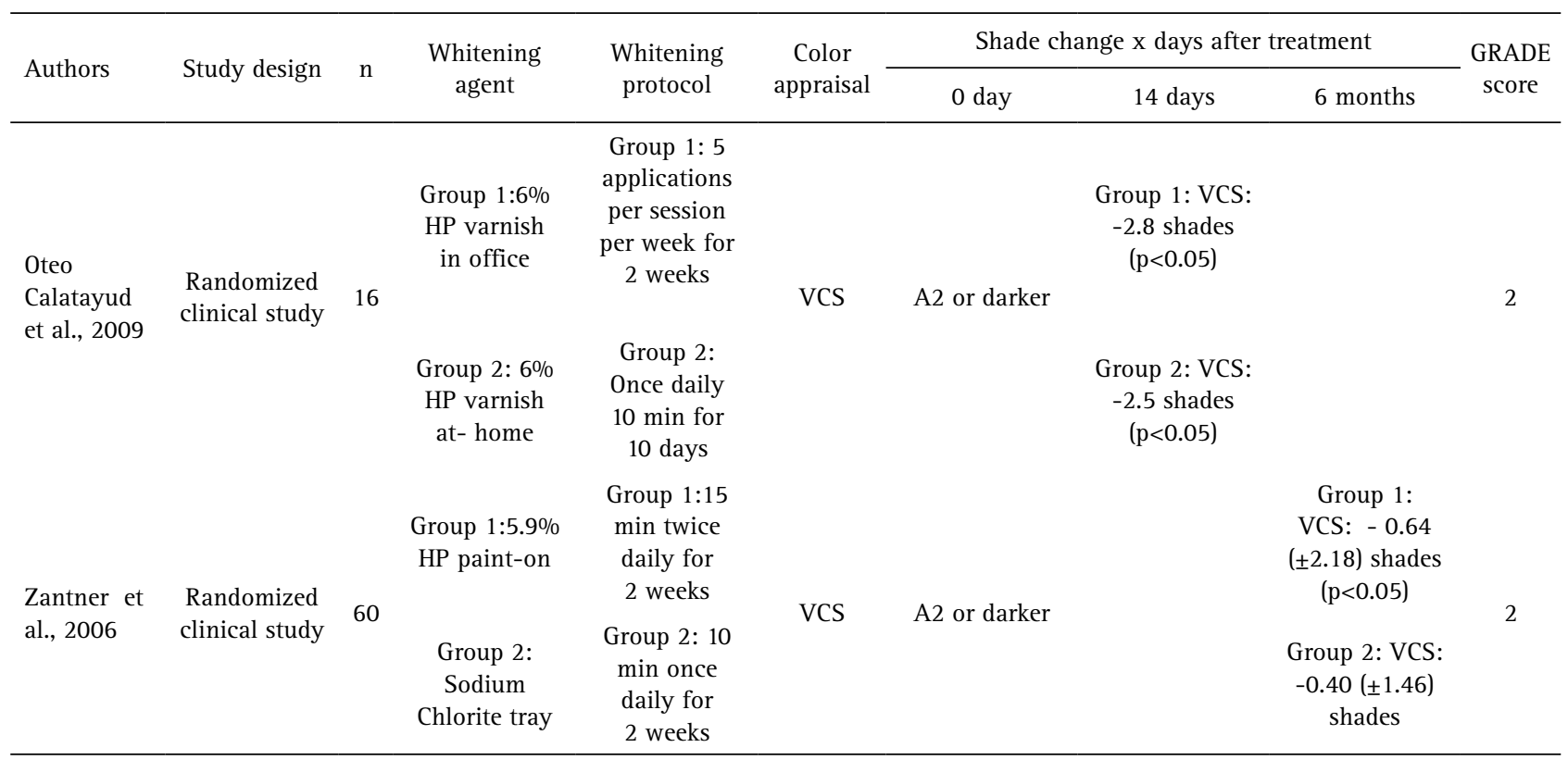

HP: Hydrogen peroxide. VCS: Vitapan Classical Shade Guide. $\triangle$ VCS: Change in tooth shade according to VCS. 
easily available to the public and low-cost. Three studies were of good quality and the remaining 7 were of lower quality (GRADE from 0 to 1). Many of the clinical studies used randomization and there was a mix of double blinded and single blinded studies. There were no set whitening or abrasive components, with some containing, baking soda, papain, hydroxyapatite, calcium carbonate and sodium fluoride. Two studies were identified that had blue covarine as a tooth-whitening agent. Blue covarine is a pigment that is uniformly deposited and retained on pellicle coated tooth surfaces. It is claimed to cause a color shift from yellow to blue, creating an illusion of tooth whitening $(22,23)$. These studies were not included in the review, as blue covarine does not remove stains or break down pigments and it merely adds a layer to create a lightening effect.

Some studies stated that the new dentifrices containing baking soda, peroxide, amorphous calcium phosphate ("active calcium") and tetrasodium pyrophosphate were effective whitening agents (24-26). Experimental dentifrices claimed to have some effectiveness with new contents such as in enzymatic dentifrices containing lactoperoxidase and carbamide peroxide (27), dual silica system and dentifrices containing sodium hexametaphosphate $(10,28,29)$. However, objective measurements did not show effectiveness in a trial carried out with hydroxyapatite and calcium peroxide for removal of extrinsic stains (30).

There was one lower quality study that reported that whitening dentifrices were not effective in changing tooth shade (31). There were 2 studies of good quality that determined that dentifrices are effective "by removing extrinsic stains" in comparison to a placebo. When dentifrices are compared to tooth whitening strips (Box 4), their effectiveness is not comparable. Yudhira et al, compared whitening dentifrice to whitening strips and determined that significant changes were seen in the strip group, but not with the dentifrice group (12).

\section{Whitening Strips}

In this review, 7 studies looked into whitening strips comparing them with either a placebo, carbamide peroxide tray, or whitening strips of different concentration. Five studies were randomized clinical studies, and five studies used hydrogen peroxide concentration in the range of 5 to $6.5 \%$ and the 2 remaining studies used high concentrations of hydrogen peroxide (9.5-14\%). The length of the studies varied from 14 days to 18 months with a standard exposure time of 30 min twice daily in all studies (Box 2). The shade taking environments were not well described and the usage of color correction lights was not specified in most studies. There was inconsistency in the studies with some not providing adequate details of the shade taking protocols. The majority of studies used a spectrophotometer.
Two studies showed that whitening strips versus placebos strips produced a significant improvement in shade $(14,32)$. Four studies reported that strips were effective and comparable to carbamide peroxide tray treatment (33-36). One study reported that whitening strip at $6 \%$ hydrogen peroxide showed superior effectiveness compared to $10 \%$ carbamide peroxide in gel tray treatment (33). A similar result was seen in a previous review that reported that strips at $6 \%$ hydrogen peroxide were more effective than $10 \%$ carbamide peroxide (6). It is interesting to note that the 2 studies that used whitening strips with $6 \%$ hydrogen peroxide and $10 \%$ carbamide peroxide used an application protocol of $30 \mathrm{~min}$ twice a day. This is not the typical application protocol for carbamide peroxide which is typically $2-4 \mathrm{~h}$ per day or overnight $(35,36)$. This would most likely severely affect the ability of the carbamide peroxide products performance. A systematic review and meta-analysis reported that there was no significant difference between whitening strips and 10\% carbamide peroxide gel whitening treatment regime (37). Furthermore, a systematic review by Luque-Martinez determined that carbamide peroxide tray treatment showed slightly better efficacy compared to whitening strips (38).

Two good quality studies showed that whitening strips were effective and that increased exposure time gave better results $(13,14)$. Oliveira et al. $(13)$ determined that a lower hydrogen peroxide concentration on strip with an extended application time showed better effectiveness than a strip with a higher hydrogen peroxide concentration applied for a shorter time. This is an interesting observation and illustrates the rate at which the peroxide is released is a factor to consider. Carbamide peroxide tray gels are slow release whereby only $50 \%$ of its peroxide is released in the first 2-4hrs and the rest over 2-6hrs, hence allowing better oxidation of the organic matrix (38). Therefore, the peroxide concentration and release rate are essential, as well as the duration of exposure (36). When compared to other OTC tooth whitening agents (Box 4), whitening strips have been seen to be effective in the majority of the studies.

\section{Paint-on Varnish/Gels}

There were only 2 studies included in this category of tooth whitening agent. Both were randomized clinical studies and used Vita Classic shade guides. Both studies concluded to varying degrees that paint-on gels were an effective means of tooth whitening $(6,18)$. Oteo Calatayud et al. (18) also concluded that there was no difference in the effectiveness if the application of paint-on varnish when done at home or in an office. While effective changes were reported by Oteo Calatayud et al. (18), Zantner et al. (20) found was little shade change after 6 months. The result was attributed to the fact that patients were advised to close 
their mouth immediately after application as per directed by the manufacturer's instructions. It pointed out that previous studies that had shown effectiveness allowed 30 $\mathrm{s}$ for the gel to dry before closing. A systematic review (6) stated paint-on gels applied three times a day were more effective than twice daily. This study (6) also stated that paint-on gels at a higher percentage of hydrogen peroxide were more effective than lower percentage gels, which is contradictory to the results of Oteo Calatayud et al. (18). Although Oteo Calatayud et al. (18) reported effectiveness of paint-on gels, it is difficult to declare paint-on gels as effective based on the limited evidence.

Box 4. Description of clinical studies that compared two or more OTC whitening agents

\begin{tabular}{|c|c|c|c|c|c|}
\hline Authors & Study design & $\mathrm{n}$ & Whitening agent & Whitening protocol & $\begin{array}{c}\text { Shade } \\
\text { appraisal }\end{array}$ \\
\hline \multirow{3}{*}{ Bizhang et al., 2007} & \multirow{3}{*}{$\begin{array}{l}\text { Randomized } \\
\text { controlled study }\end{array}$} & \multirow{3}{*}{71} & $\begin{array}{c}\text { Group 1: } \\
\text { 6\% HP whitening } \\
\text { strips }\end{array}$ & $\begin{array}{c}\text { Group 1: } \\
30 \text { min twice daily } \\
\text { for } 2 \text { weeks }\end{array}$ & \multirow{3}{*}{ S } \\
\hline & & & $\begin{array}{c}\text { Group 2: } \\
\text { 19\% sodium } \\
\text { percarbonate brush } \\
\text { applied gel }\end{array}$ & $\begin{array}{l}\text { Group 2: } \\
1 \text { Brush application } \\
\text { daily for } 14 \text { nights }\end{array}$ & \\
\hline & & & $\begin{array}{l}\text { Group 3: } \\
\text { Placebo without } \\
\text { peroxide }\end{array}$ & $\begin{array}{c}\text { Group 3: } \\
1 \text { Brush application } \\
\text { daily for } 14 \text { nights }\end{array}$ & \\
\hline \multirow{3}{*}{ Bizhang et al., 2009} & \multirow{3}{*}{$\begin{array}{l}\text { Clinical } \\
\text { study }\end{array}$} & \multirow{3}{*}{75} & $\begin{array}{c}\text { Group 1: } \\
\text { 6\% HP whitening } \\
\text { strips }\end{array}$ & $\begin{array}{l}\text { Group 1: } \\
30 \text { min twice daily } \\
\text { for } 2 \text { weeks }\end{array}$ & \multirow{3}{*}{ S } \\
\hline & & & $\begin{array}{c}\text { Group 2: } \\
\text { 10\% CP tray at home }\end{array}$ & $\begin{array}{c}\text { Group 2: } \\
\text { Overnight for } 2 \text { week }\end{array}$ & \\
\hline & & & $\begin{array}{l}\text { Group 3: } \\
15 \% \text { HP in office }\end{array}$ & $\begin{array}{c}\text { Group3: } \\
45 \text { min once daily, } \\
3 \text { times for } 3 \text { weeks }\end{array}$ & \\
\hline \multirow{3}{*}{$\begin{array}{l}\text { Lo } \\
\text { et al., } 2007\end{array}$} & \multirow{3}{*}{$\begin{array}{l}\text { Randomized } \\
\text { controlled study }\end{array}$} & \multirow{3}{*}{63} & $\begin{array}{l}\text { Group 1: 6\% HP } \\
\text { whitening strips }\end{array}$ & $\begin{array}{c}\text { Group 1: } \\
30 \text { min twice daily } \\
\text { for } 2 \text { weeks }\end{array}$ & \multirow{3}{*}{$\mathrm{S}$} \\
\hline & & & $\begin{array}{l}\text { Group 2: } \\
\text { 18\% CP paint-on gel }\end{array}$ & $\begin{array}{c}\text { Group 2: } \\
15 \text { min twice daily } \\
\text { for } 2 \text { weeks }\end{array}$ & \\
\hline & & & $\begin{array}{c}\text { Group 3: } \\
\text { Placebo (strips and } \\
\text { 6\% Fluoride) }\end{array}$ & $\begin{array}{l}\text { Group 3: } \\
30 \text { min twice daily } \\
\text { for } 2 \text { weeks }\end{array}$ & \\
\hline \multirow{3}{*}{ Yudhira et al., 2007} & \multirow{3}{*}{$\begin{array}{l}\text { Randomized } \\
\text { controlled study }\end{array}$} & \multirow{3}{*}{46} & $\begin{array}{c}\text { Group 1: } \\
\text { 6\% HP whitening } \\
\text { strips }\end{array}$ & $\begin{array}{c}\text { Group 1: } \\
30 \text { min twice daily } \\
\text { for } 2 \text { weeks }\end{array}$ & \multirow{3}{*}{$S$} \\
\hline & & & $\begin{array}{l}\text { Group 2: Whitening } \\
\text { toothpaste } \mathrm{NaF}\end{array}$ & $\begin{array}{l}\text { Group 2: } \\
\text { Twice daily for } \\
12 \text { weeks }\end{array}$ & \\
\hline & & & $\begin{array}{c}\text { Group 3: } \\
\text { Whitening toothpaste } \\
\text { MFP }\end{array}$ & $\begin{array}{l}\text { Group 3: } \\
\text { Twice daily for } \\
12 \text { weeks }\end{array}$ & \\
\hline \multirow{3}{*}{ Xu Xiao et al., 2007} & \multirow{3}{*}{$\begin{array}{l}\text { Randomized } \\
\text { controlled study }\end{array}$} & \multirow{3}{*}{49} & $\begin{array}{c}\text { Group 1: } \\
\text { 6\% HP whitening } \\
\text { strips }\end{array}$ & $\begin{array}{l}\text { Group 1: } \\
\text { Twice daily } \\
\text { for } 1 \text { week }\end{array}$ & \multirow{3}{*}{$\mathrm{S}$} \\
\hline & & & $\begin{array}{c}\text { Group 2: } \\
\text { 5.9\% HP paint-on gel }\end{array}$ & $\begin{array}{l}\text { Group 2: } \\
\text { Twice daily } \\
\text { for } 2 \text { weeks }\end{array}$ & \\
\hline & & & $\begin{array}{c}\text { Group 3: } \\
\text { Placebo } \\
\text { (water rinse) }\end{array}$ & $\begin{array}{l}\text { Group 3: } \\
\text { Twice daily } \\
\text { for } 2 \text { weeks }\end{array}$ & \\
\hline
\end{tabular}




\section{Comparison of Different Over-The-Counter Tooth Whitening Agents}

In this review several studies compared two or more OTC tooth whitening agents allowing comparison. There were 5 studies included in this category, out of which 4 were of good quality (Box 4) which reported the superior effectiveness of whitening strip compared to other OTC tooth whitening agents $(12,15,17,18)$.

In general whitening strips showed superior effectiveness when compared to other OTC tooth whitening agents such as mouth rinses, dentifrices and paint-on gels. This was contributed to the higher contact time and the

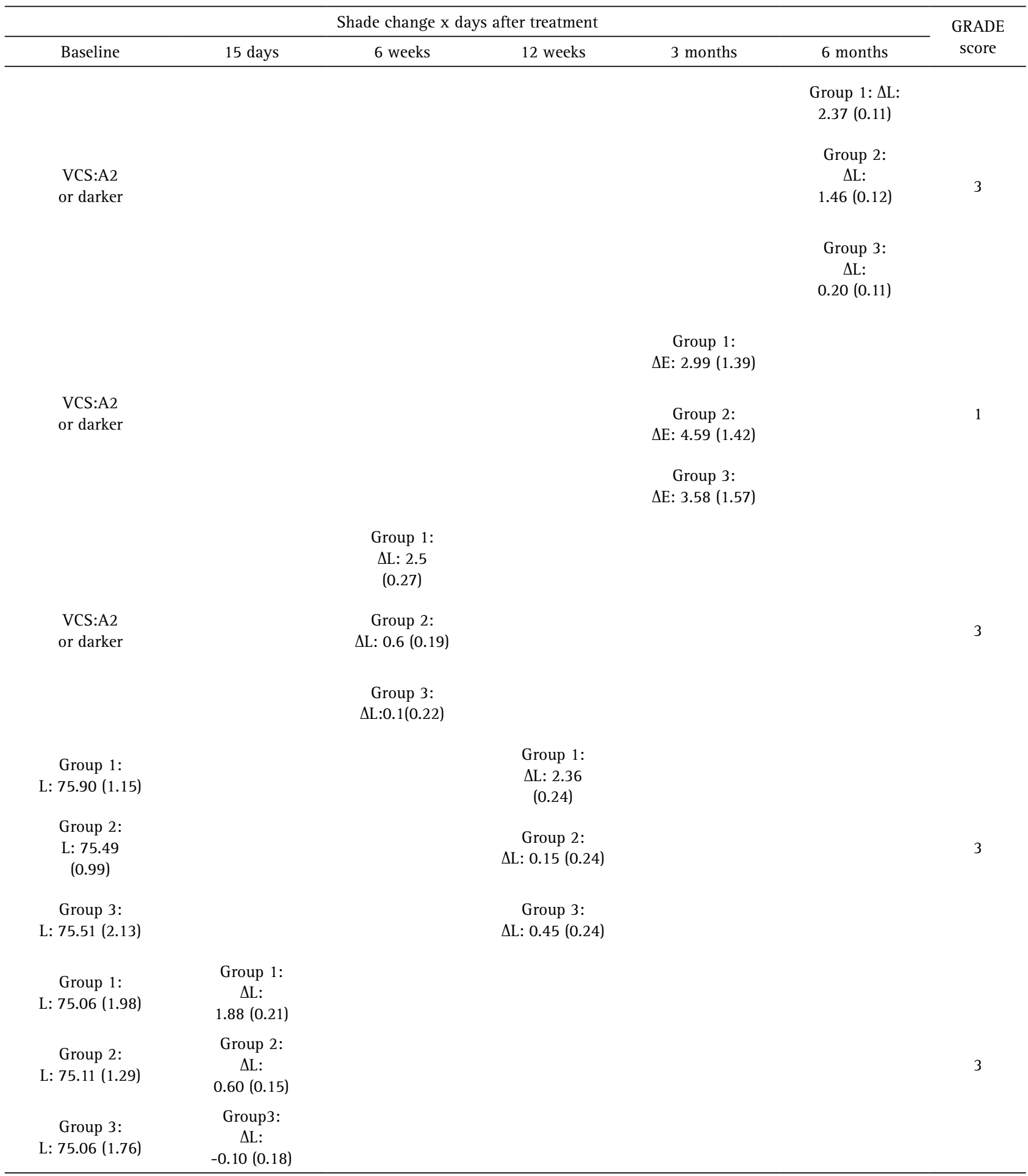


concentration of hydrogen peroxide of whitening strips. The difference between paint-on gels and whitening strips effectiveness is attributed to the fact that the strip acts as a fixed barrier (reservoir) unlike paint-on gels (15). All studies were randomized according to age and the color of teeth. From this review it could be concluded that when comparing with OTC tooth whitening agents, whitening strips have consistently been proven to have superior effectiveness.

Within the limitations of this study and from the evidence, it can be concluded that with regards to the effectiveness of OTC tooth whitening agents:

Whitening strips are an effective OTC tooth-whitening agent when compared to a placebo, other OTC tooth whitening agents, however their effectiveness compared to tray-based gels with carbamide peroxide is debatable and dependent on the carbamide peroxide percentage and the application timespan.

Whitening dentifrices are effective in changing the shade of the tooth "by removing extrinsic stains" when compared to a placebo and non-whitening dentifrices. They are not as effective when compared with tooth whitening strips.

3. There is a lack of evidence to make a conclusion with regards the effectiveness of paint-on gels whitening agents.

There is a need for more independent standardized clinical studies and randomized clinical studies in the area of OTC products effectiveness.

The majority of the studies were conducted over a short time span, which may not represent the true effectiveness of the product tested. The aspect of relapse therefore would be an area for further research.

\section{Resumo}

Há um aumento da acessibilidade dos agentes clareadores de venda livre com poucos dados na literatura sobre sua eficácia. Esta revisão foi feita para determinar a eficácia dos agentes clareadores de venda livre predominantes de 2006 a 2018, onde foi feita uma comparação de cada agente com um placebo, sem tratamento ou com outros agentes clareadores de venda livre. As principais categorias de agentes clareadores de venda livre, como dentifrícios, tiras branqueadoras e géis. Estudos em que o dentista prescreveu clareamento caseiro e em consultório, e estudos que demonstraram os agentes clareadores para os participantes. Os artigos foram pesquisados nas bases de dados do Medline (Ovid), PubMed, Cochrane Library e Cochrane Central Register of Controlled Trials. Vinte e quatro artigos foram incluidos na revisão sistemática e a qualidade dos estudos foi determinada pelo critério de classificação GRADE (Classificação de Recomendações, Avaliação, Desenvolvimento e Avaliações). Em comparação com outros clareadores de venda livre, as tiras são relatadas como eficazes. Dois estudos determinaram que as tiras de clareamento são eficazes. As tiras de clareamento mostraram-se eficazes quando comparadas com placebos e outros agentes clareadores de venda livre. Os dentifrícios são eficazes na mudança da tonalidade do dente "removendo manchas extrínsecas" quando comparados a dentifrícios com placebo e sem clareamento, mas não são tão eficazes em comparação com as tiras de clareamento. Há uma falta de evidência com relação à eficácia dos géis de tinta. Embora exista alguma evidência de que os clareadores de venda livre possa alterar a tonalidade no curto prazo, há necessidade de estudos melhor projetados.

\section{Acknowledgements}

The Authors appreciate the support from the Sir John Walsh Research Institute and thank the Faculty of Dentistry, University of Otago, and New Zealand for the use of their facilities. The authors would like to thank $\mathrm{Mr}$ Andrew Grey for the statistical analysis.

\section{References}

1. Joiner A. The bleaching of teeth: A review of the literature. J Dent 2006;34:412-419.

2. Kugel G. Over the counter tooth-whitening systems. Compend Contin Educ Dent 2003;24:376-382.

3. Carey CM. Tooth whitening: What we now know. J Evid Based Dent Pract 2014;14:70-76.

4. Demarco FF, Meireles SS, Masotti AS. Over-the-counter whitening agents: A concise review. Braz Oral Res 2009;23:64-70.

5. Collins LZ, Maggio B, Liebman J, Blanck M, Lefort S, Waterfield P, et al. Clinical evaluation of a novel whitening gel, containing $6 \%$ hydrogen peroxide and a standard fluoride toothpaste. J Dent 2004;32:13-17.

6. Hasson H, Ismail Al, Neiva G. Home-based chemically-induced whitening of teeth in adults. Cochrane Database Syst Rev 2006;Cd006202.

7. Guyatt GH, Oxman AD, E Vist G, Kunz R, Falck- Ytter Y, Alonso-Coello $P$, et al. GRADE: an emerging consensus on rating quality of evidence and strength of recommendations. BMJ 2008;336:924.

8. Guyatt GH, Oxman AD, AkI EA, Kunz R, Vist G, Brozek J, et al. GRADE guideline: 1.Introduction - GRADE evidence profiles an summary of findings tables. J Clin Epidemiol 2011;64:383-394.

9. Liberati A, Altman DG, Tetzlaff J, Mulrow C, Gotzsche PC, loannidis JP et al. The PRISMA statement for reporting systematic reviews and metaanalyses of studies that evaluate health care interventions: explanation and elaboration. J Clin Epidemiol 2009;62:e1-34.

10. Terezhalmy G, Chaves E, Bsoul S, Baker R, He T. Clinical evaluation of the stain removal efficacy of a novel stannous fluoride and sodium hexametaphosphate dentifrice. Am J Dent 2007;20:53-58.

11. Young S, Parkinson C, Hall C, Wang N, Milleman JL, Milleman KR. A randomized clinical study investigating the stain-removal potential of two experimental dentifrices. J Clin Dent 2015;26:96-103.

12. Yudhira R, Peumans $M$, Barker ML, Gerlach RW. Clinical trial of tooth whitening with $6 \%$ hydrogen peroxide whitening strips and two whitening dentifrices. Am J Dent 2007;20:32A-36A.

13. Oliveira GM, Miguez PA, Oliveira GB, Swift EJ Jr., Farrell S, Anastasia MK, et al. Safety and efficacy of a high-adhesion whitening strip under extended wear regimen. J Dent 2013;1:e46-e52.

14. Swift EJ Jr, Heymann HO, Wilder AD Jr, Barker ML, Gerlach RW. Effects of duration of whitening strip treatment on tooth color: A randomized, placebo-controlled clinical trial. J Dent 2009;37:e51-e56.

15. Bizhang $M$, Muller M, Phark JH, Barker ML, Gerlach RW. Clinical trial of long-term color stability of hydrogen peroxide strips and sodium percarbonate film. Am J Dent 2007;20:23A-27A.

16. Bizhang $M$, Chun $Y H$, Damerau $K$, Singh $P$, Raab WH, Zimmer $S$. Comparative clinical study of the effectiveness of three different bleaching methods. Oper Dent 2009;34:635-641.

17. Xu X, Zhu L, Tang Y, Wang Y, Zhang K, Li S, et al. Randomized clinical trial comparing whitening strips, paint-on gel and negative control. Am J Dent 2007;20:28-31.

18. Lo EC, Wong AH, McGrath $\mathrm{C}$. A randomized controlled trial of home tooth-whitening products. Am J Dent 2007;20:315-318.

19. Oteo Calatayud J, Mateos de la Varga P, Oteo Calatayud C, Calvo Box MJ. 2009. Comparative clinical study of two tooth bleaching protocols with 6\% hydrogen peroxide. Int J Dent 2009:928306.

20. Zantner C, Derdilopoulou F, Martus P, Kielbassa AM. Randomized clinical trial on the efficacy of 2 over-the-counter whitening systems. 
Quintessence Int 2006:37:695-706.

21. Chu SJ, Trushkowsky RD, Paravina RD. Dental color matching instruments and systems. Review of clinical and research aspects. J Dent. 2010;38:e2-e16.

22. Collins $L Z$, Naeeni M, Platten SM. Instant tooth whitening from a silica toothpaste containing blue covarine. J Dent 2008;36:S21-S25.

23. Dantas $\mathrm{AA}$, Bortollatto JF, Roncolato $\mathrm{A}$, Merchan $\mathrm{H}$, Floros $M C$, Kuga $M C$, et al. Can a bleaching toothpaste containing blue covarine demonstrate the same bleaching as conventional techniques? An in vitro, randomized and blinded study. J Appl Oral Sci 2015;23:609-613.

24. Ghassemi A, Vorwerk L, Cirigliano A, Hooper W, DeSciscio P, Nathoo S. Clinical effectiveness evaluation of a new whitening dentifrice. J Clin Dent 2015a;26:66-71.

25. Ghassemi A, Hooper W, Vorwerk L, Domke T, DeSciscio P, Nathoo S. Effectiveness of a new dentifrice with baking soda and peroxide in removing extrinsic stain and whitening teeth. J Clin Dent 2012;23:8691.

26. Ghassemi A, Vorwerk L, Hooper W, Cirigliano A, DeSciscio P, Nathoo S. Extrinsic stain removal effectiveness of a new whitening dentifrice. J Clin Dent 2015;26:72-75.

27. Forner L, Amengual J, Liena C, Riutord P. Therapeutic effectiveness of a new enzymatic bleaching dentifrice. Eur J Esthet Dent 2012;7:62-70.

28. Nathoo S, Singh S, Petrone DM, Wachs GN, Chaknis P, DeVizio W, et al. Clinical studies to assess the extrinsic stain prevention and stain removal efficacy of a variant of a commercially available dentifrice containing a new dual silica system. J Clin Dent 2008;19:95-101.

29. Terezhalmy GT, Biesbrock AR, Farrell S, Barker ML, Bartizek RD. Tooth whitening through the removal of extrinsic stain with two sodium hexametaphosphate-containing whitening dentifrices. Am J Dent 2007;20:309-314.

30. Raoufi S, Birkhed D. Effect of whitening toothpastes on tooth staining using two different color-measuring devices-a 12-week clinical trial.
Int Dent J 2010;60:419-423.

31. Horn BA, Bittencourt BF, Gomes OM, Farhat PA. Clinical evaluation of the whitening effect of over-the-counter dentifrices on vital teeth. Braz Dent J 2014;25:203-206.

32. Guerrero JC, Jimenez-Farfan MD, Lopez-Salgado A, Barker ML, Gerlach RW. Professional whitening strips in a university population. Am J Dent 2007;20:15A-18A.

33. Auschill TM, Schneider-Del Savio T, Hellwig E, Arweiler NB. Randomized clinical trial of the efficacy, tolerability, and long-term color stability of two bleaching techniques: 18-month follow-up. Quintessence Int 2012;43:683-694.

34. da Costa JB, McPharlin R, Hilton T, Ferracane JI, Wang M. Comparison of two at-home whitening products of similar peroxide concentration and different delivery methods. Oper Dent 2012;37:333-339.

35. Ferrari M, Cagidiaco MC, Monticelli F, Kugel G, Barker ML, Gerlach RW. Daytime use of a custom bleaching tray or whitening strips: Initial and sustained color improvement. Am J Dent 2007;20:19A-22A.

36. Hannig $C$, Lindner D, Attin T. Efficacy and tolerability of two home bleaching systems having different peroxide delivery. Clin Oral Investig 2007;11:321-329.

37. Serraglio CR, Zanella L, Dalla-Vecchia KB, Rodrigues-Junior SA. Efficacy and safety of over-the-counter whitening strips as compared to homewhitening with 10\% carbamide peroxide gel--systematic review of RCTs and metanalysis. Clin Oral Investig 2016;20:1-14.

38. Luque-Martinez I, Reis $A$, Schroeder M, Munoz MA, Loguercio AD, Masterson D, et al. Comparison of efficacy of tray-delivered carbamide and hydrogen peroxide for at-home bleaching: A systematic review and meta-analysis. Clin Oral Investig 2016;20:1419-1433.

Received November 1, 2019 Accepted January 31, 2020 